\title{
System Calibration and Statistical Image Reconstruction for Ultra-High Resolution Stationary Pinhole SPECT
}

\author{
Frans van der Have*, Member, IEEE, Brendan Vastenhouw, Mart Rentmeester, and \\ Freek J. Beekman, Senior Member, IEEE
}

\begin{abstract}
For multipinhole single-photon emission computed tomography (SPECT), iterative reconstruction algorithms are preferred over analytical methods, because of the often complex multipinhole geometries and the ability of iterative algorithms to compensate for effects like spatially variant sensitivity and resolution. Ideally, such compensation methods are based on accurate knowledge of the position-dependent point spread functions (PSFs) specifying the response of the detectors to a point source at every position in the instrument. This paper describes a method for model-based generation of complete PSF lookup tables from a limited number of point-source measurements for stationary SPECT systems and its application to a submillimeter resolution stationary small-animal SPECT system containing 75 pinholes (U-SPECT-I). The method is based on the generalization over the entire object to be reconstructed, of a small number of properties of point-source responses which are obtained at a limited number of measurement positions. The full shape of measured point-source responses can almost be preserved in newly created PSF tables. We show that these PSFs can be used to obtain high-resolution SPECT reconstructions: the reconstructed resolutions judged by rod visibility in a micro-Derenzo phantom are $0.45 \mathrm{~mm}$ with 0.6-mm pinholes and below $0.35 \mathrm{~mm}$ with $0.3-\mathrm{mm}$ pinholes. In addition, we show that different approximations, such as truncating the PSF kernel, with significant reduction of reconstruction time, can still lead to acceptable reconstructions.
\end{abstract}

Index Terms-Calibration, pinhole, reconstruction, singlephoton emission computed tomography (SPECT).

\footnotetext{
Manuscript received January 30, 2008; revised April 10, 2008. This work was supported by the Netherlands Organization for Scientific Research (NWO) under Grant 917.36.335. Asterisk indicates corresponding author.

*F. van der Have is with the Department of Nuclear Medicine, Image Sciences Institute, and the Department of Pharmacology and Anatomy, Rudolf Magnus Institute of Neuroscience, University Medical Center Utrecht, 3584 CG Utrecht, The Netherlands, and with the Milabs B.V., 3584 Utrecht, The Netherlands, and also with the Section Radiation Detection and Matter, Delft University of Technology, Mekelweg 15 Delft, The Netherlands (e-mail f.vanderhave@umcutrecht.nl).

B. Vastenhouw and M. Rentmeester are with the Department of Nuclear Medicine, Image Sciences Institute, and the Department of Pharmacology and Anatomy, Rudolf Magnus Institute of Neuroscience, University Medical Center Utrecht, 3584 CG Utrecht, The Netherlands.

F. J. Beekman is with the Department of Nuclear Medicine, Image Sciences Institute, and the Department of Pharmacology and Anatomy, Rudolf Magnus Institute of Neuroscience, University Medical Center Utrecht, 3584 CG Utrecht, The Netherlands, and with the Milabs B.V., 3584 Utrecht, The Netherlands, and also with the Section Radiation Detection and Matter, Delft University of Technology, Mekelweg 15 Delft, The Netherlands.

Color versions of one or more of the figures in this paper are available online at http://ieeexplore.ieee.org.

Digital Object Identifier 10.1109/TMI.2008.924644
}

\section{INTRODUCTION}

$\mathbf{S}$ INGLE-PHOTON emission computed tomography (SPECT) permits in vivo volumetric imaging of 3-D distributions of radio-labeled molecules. Pinhole-collimated SPECT is particularly suitable for studying laboratory animals such as mice and rats, because for small objects it can achieve both a high spatial system resolution and a high sensitivity when compared to parallel-hole collimated SPECT. Several SPECT systems using pinhole collimation have been developed [1]-[7] (for reviews see [8]-[11] and for design considerations see [12]). Stationary systems (that do not need to rotate their detectors) with tens up to hundreds of pinholes have been designed [13]-[17]. A high number of pinholes results in a high sensitivity which can be traded for a higher system resolution by decreasing the pinhole diameter. The stationary designs provide excellent stability and unique capabilities to perform fast dynamic studies, e.g., [13], [18], [19].

Today, iterative reconstruction algorithms are prominent in SPECT. Iterative algorithms have the advantage that they can benefit from accurate models of the photon transport in the SPECT system and they can handle a large variety of detector-collimator geometries. In addition, they can deal with noise better than direct methods of reconstruction. Several papers have shown that the more accurate photon transport is modeled during iterative reconstruction the more accurate the reconstructed images will be, not only in terms of resolution and quantitative accuracy but also in terms of signal-to-noise ratio and lesion detectability, e.g., [20]-[24]. An important advantage of iterative reconstruction is that no explicit mathematical expression for the inverse transformation is required. Iterative algorithms only need a way to predict the detector response to a given radio nuclide distribution in the object. To this end, the object space is most times divided into volume elements (voxels) and the total detector response is assumed to be the sum of the responses to small radioactive sources in all of the voxels. A specific detector response to a "point" source is known as the point spread function (PSF). The individual PSFs for all voxels collectively contain the entire information to set up the "system matrix" or "transition matrix" $M$ of the SPECT system. Each matrix element $M_{j i}$ represents the likelihood that a photon emitted in voxel $i$ is detected in detector pixel $j$. Then, the activity distribution $\vec{a}$ is estimated from $\vec{p}=M \cdot \vec{a}+\vec{n}$ where $\vec{p}$ is the measured projection and $\vec{n}$ represents the noise in each pixel. 


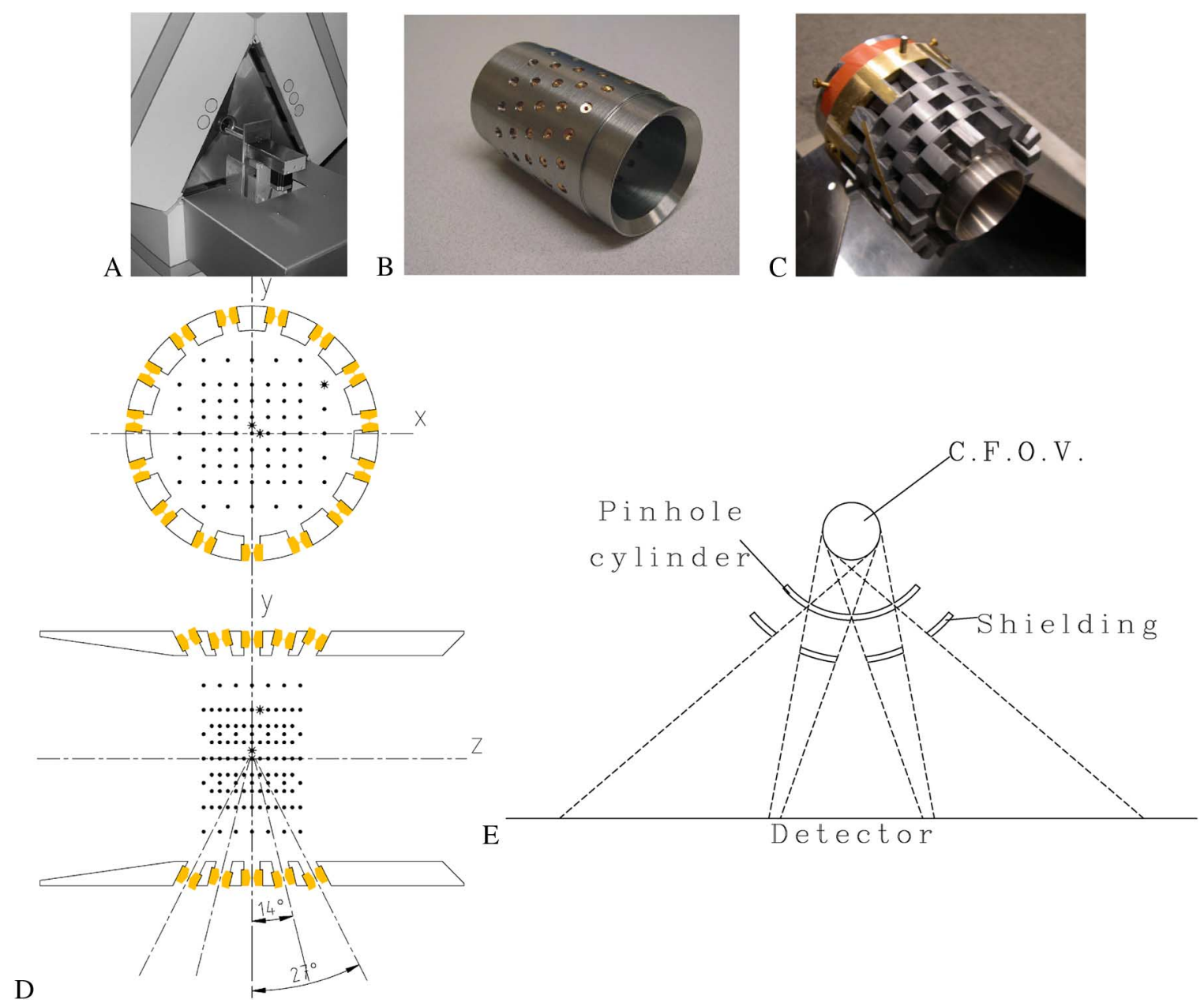

Fig. 1. A: U-SPECT-I system. Triangular-shaped lead shielding is placed in between three camera heads. A tungsten cylinder containing pinholes is placed in the center of the three detectors. An XYZ-stage with attached bed is placed in front of the lower detector. B: Cylinder with 75 gold pinhole apertures. C: Cylinder with pinholes surrounded by the shielding that prevents projections to overlap. D: Cross section of the cylinder with focusing pinholes. $x, y$, and $z$ define the coordinate system used in the object space. Solid circles indicate the positions of the point source used for calibration. Stars indicate the example point source positions for validation. E: Schematic cross section of the shielding tube that prevents projections from overlapping.

The PSFs for SPECT are sometimes calculated analytically [4], [25]-[27], given "known" properties of the system. Pinhole collimators make the detector response very sensitive to some parameters, e.g., the exact position, orientation, size, and shape of each pinhole aperture in the system. Methods for the calibration of rotating pinhole SPECT systems (based on calibration systems for cone-beam SPECT with similar geometry) have been proposed, e.g., [26], [28]-[33], that can determine the acquisition geometry (e.g., the position of the pinhole with respect to the axis of rotation and the position, tilt and twist of the detector) using one up to three point sources. These systems still use an analytical model to estimate the pixel values in a PSF. In stationary pinhole systems, it is feasible to determine the PSFs themselves experimentally with a large number of point-source measurements. This has the advantage that the actual positions, orientations, and sizes of the pinholes and detector pixel sensitivities are accurately incorporated into the PSF tables. Difficulties in manufacturing precise pinhole positions and shapes with exactly the specified diameter (which is only a few tenths of a millimeter for high-resolution SPECT in mice) could make analytically predicted PSFs deviate from reality. The approach of measuring the complete matrix with a point source was developed at the University of Arizona and was used to calibrate different SPECT systems [14], [15], [34], [35].

Recently, resolutions of small-animal SPECT systems have improved from typically a few millimeter to sub-half-millimeter resolution [11], [16], [17], [36], [37], which makes it hard to measure the PSF for each voxel: up to millions of tiny voxels are needed to represent the high-resolution images. Then, it is impractical to put a point source that is approximately the size of one voxel at every voxel position. The maximum achievable concentration of radioactivity and the number of counts that have to be acquired from every point source position, make it a prohibitively long measurement. In addition, the point source may need to be replaced many times predictably at a precisely known position. Apart from a high number of voxels, a high-resolution scanner may also need a relatively high number of pixels. This combination could mean that the intermediate storage of all raw data requires enormous amounts of disk space on systems where the acquisition hardware or software does not permit the acquired data to be directly stored in other formats than raw pixelized images. 

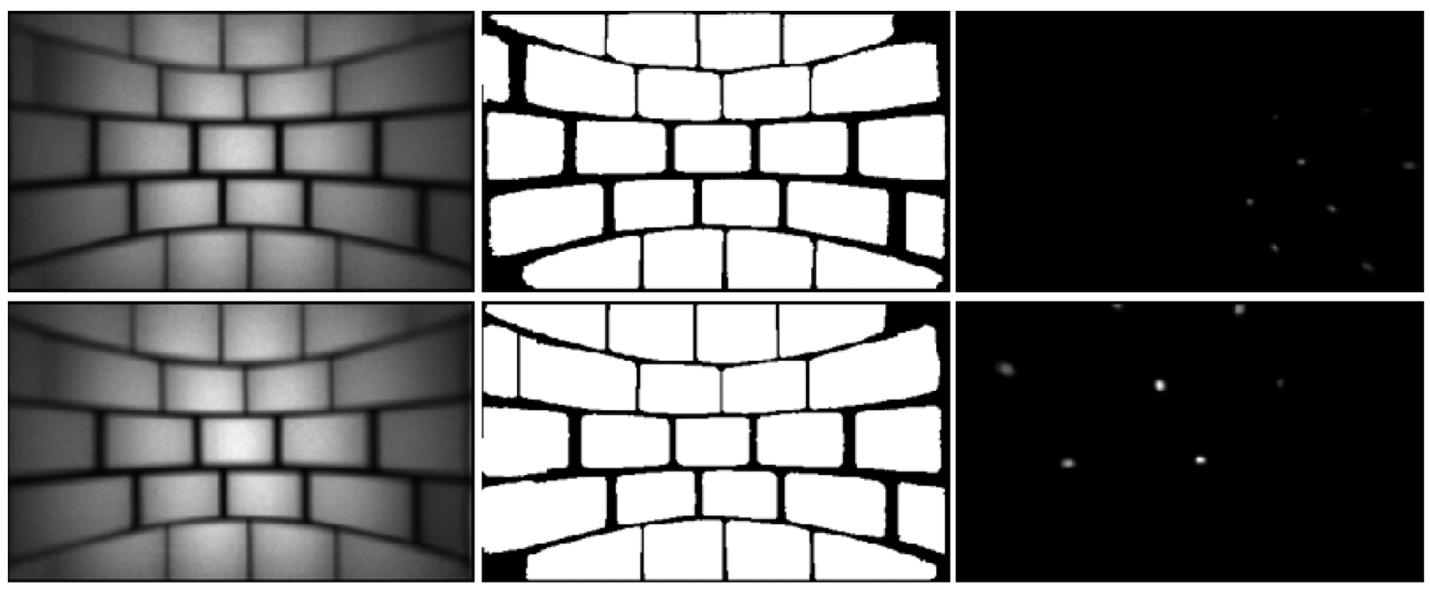

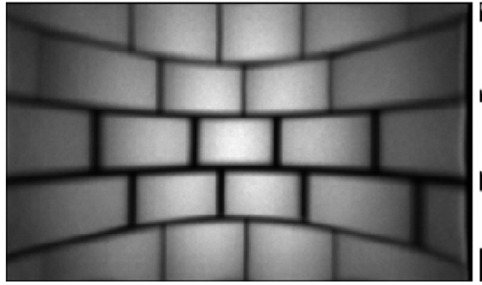

A

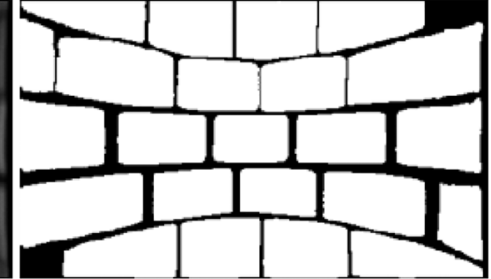

B

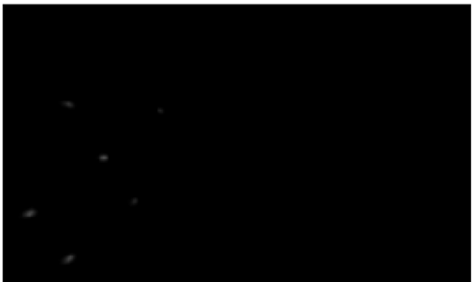

C

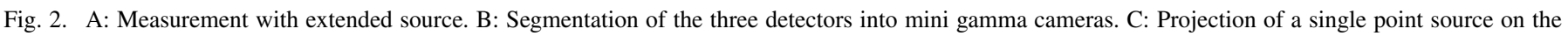
mini cameras.

Here, we propose and validate an efficient and accurate method to obtain the position-sensitive PSF tables for a high-resolution many-pinhole system [16], [17]. We explain how the full system matrix can be determined from measurements at a limited number of positions. In addition, we will present examples to give an impression of the accuracy of estimated PSFs as well as reconstructed images. The trade-off between truncation of the PSF kernel and the reconstruction speed was also investigated. Reconstructed images are presented for a capillary resolution phantom with different numbers of acquired counts.

\section{METHODS}

The method described in this paper is developed for the stationary pinhole SPECT systems U-SPECT-I and U-SPECT-II [16], [36], [38]. These systems have 75 focusing pinholes in a cylindrical configuration with five rings of 15 pinholes each (see Fig. 1). The collimator cylinder is placed in the center of three gamma camera heads. Each of the camera heads is divided into a large set of mini gamma cameras, each dedicated to the projection through one pinhole. We ensure that the projection areas are nonoverlapping by means of a shielding tube [16], [39], [40]. Fig. 2 shows how this results in dividing the three large detectors in many small cameras. It also shows an image obtained by placing a bottle with Tc- $99 \mathrm{~m}$ pertechnetate in the scanner as well as an image from a point source.

The method described here for obtaining the PSF tables is based on calculating a number of defining properties at the detector segments (namely the position on the detector, the flux, and the spatial extent) for each measured point-source response. The available data are then used to fit a parametric model of these properties and the fit results are used to predict the PSFs for all voxels.

Full PSF tables (representing the "system matrix") are obtained out of a set of point-source responses in four steps.

Step 1) Noise suppression and identification of areas that contain the local maximum of a point-source response.

Step 2) Characterizing the PSFs using Gaussian modeling.

Step 3) Generalization of the PSF's model over the object space using an analytical pinhole model.

Step 4) Calculation of the supplementary PSFs for storage in tables.

These steps are explained in more detail in the subsections below.

\section{A. Noise Suppression and Identification of Areas That Contain the Local Maximum of a Point-Source Response}

High-frequency noise in the point-source projections is suppressed by a fit procedure that is based on fitting Gaussian basis functions with a certain width to the data using a maximum likelihood expectation maximization (ML-EM) algorithm. This is equivalent to Richardson-Lucy deconvolution [41], [42] followed by one convolution with the same Gaussian kernel. More details about the procedure can be found in Colijn et al. [43]. In contrast to low-pass filtering, this procedure does not degrade the resolution of the measurements, since the width of the convolution kernel [3.0 mm full-width at half-maximum (FWHM)] is chosen slightly smaller than the detectors' intrinsic resolution (3.2 mm FWHM). The result of the procedure is demonstrated in Fig. 3.

Each detector segment should contain either one or no projected point source. The "worst case" situation resulting in the 

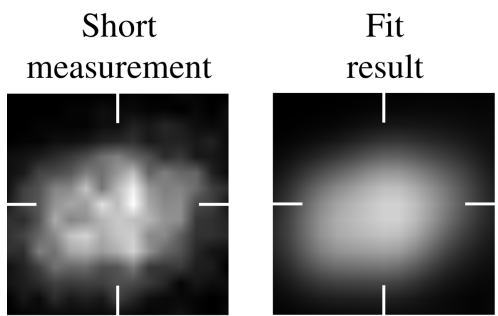

Long

measurement

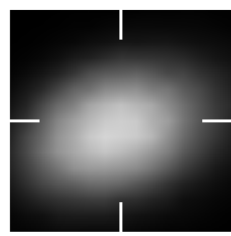

horizontal profile (1 pixel)
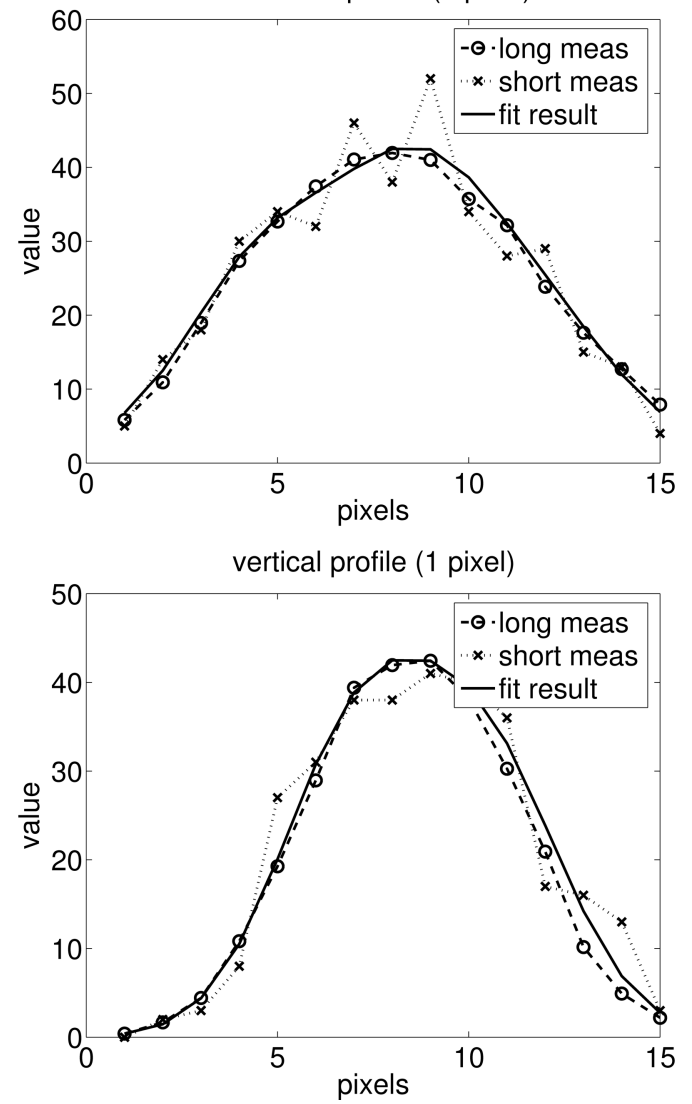

Short

measurement
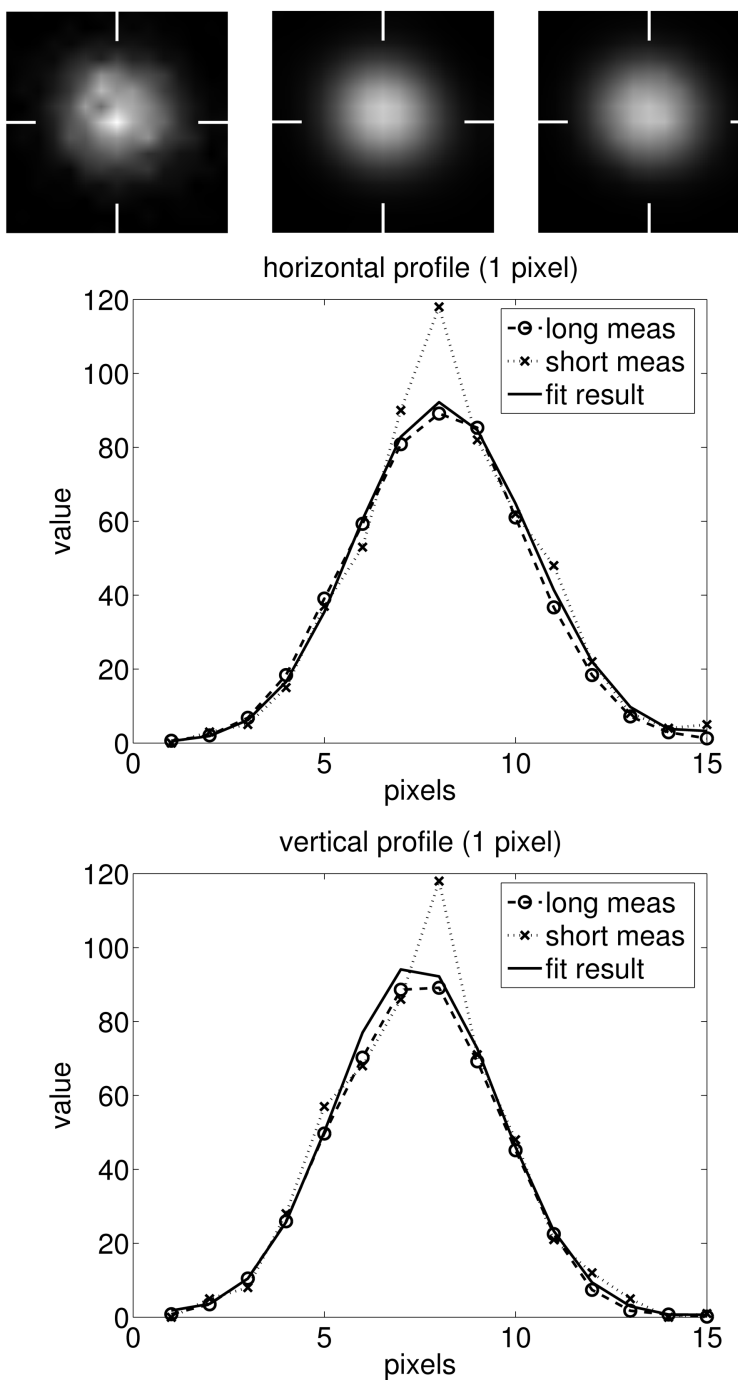

Fig. 3. Effect of the noise-suppression procedure for two point-source projections. Image after fitting is close to a reference image that has a 40 times longer acquisition period. Horizontal and vertical profiles show the values of a single row and column of pixels through the maximum, indicated by white markers.

lowest number of counts per pixel is considered. That is when the point source is located at the measurement position that is the farthest away from the pinhole and at the maximum angle from the pinhole's axis. Based on the maximum pixel value in that situation, a threshold level is chosen. Only those detector segments where at least one pixel is above this local threshold are assumed to contain a PSF.

\section{B. Characterizing the PSFs Using Gaussian Modeling}

As the next step, each measured point-source response is divided pixel-by-pixel by a high-count projection image of a cylindrical reservoir (bottle) that was filled homogeneously with Tc-99m. To avoid undesirable truncation effects the bottle is a few times larger than the central field-of-view of the system. The high-count bottle projection contains information about the partial shielding of pixels at segment edges as defined by the baffles that prevent overlapping of projections.

Division by the bottle projection converts the relative intensities of neighboring edge pixels to approximately what they would have been if there were no baffles. It does not take into account scattered photons and photons that penetrate the shielding, but the relative contribution of those photons is low [44]. Fig. 4 illustrates the effect of the division. The procedure enables pixels near the segment edges to be used for reconstruction, but introduces a low-frequency change in the PSF. The division of the angular response of the pinhole by the projection of a cylindrical activity distribution may reduce the curvature of the low-frequency response, as illustrated by Fig. 4, which shows a profile with the shape of a strongly bent arc being transformed into a profile with less pronounced curvature. Later in the calibration procedure (Section II-D), the pixel values will be multiplied again by the same extended homogeneous source projection. This restores the original scaling and recovers the appropriate pixel values in the PSFs. Therefore the low-frequency change induced by the division does not end up in the final PSF tables.

After noise suppression as described in Section II-A, and after division by the high-count uniform source projection, for 

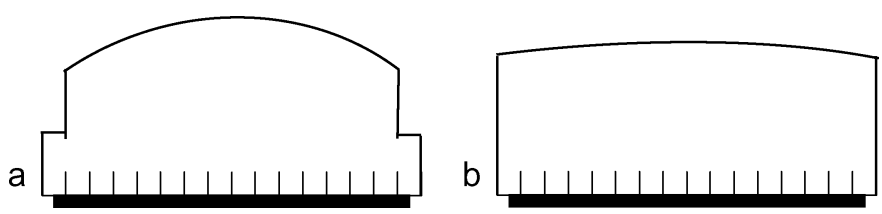

Fig. 4. Effect of the projection division by a homogeneous source image. A: Profile of the projection of an extended object before division. The part of the detector that is not blocked by the shielding, is represented by the black rectangle. The short vertical lines indicate the pixel boundaries. The outermost pixels are covered by the shielding for part of their surface, in this example half of it. B: Profile of the same object projection, but after the division.

each segment the position of the maximum on the detector $(\xi, \eta)$, the amplitude $A$, and the width $\sigma$ are estimated for each point-source measurement using the Powell fitting algorithm [45]. This is an unweighted least-squares fit of a 2-D circular Gaussian

$$
A \cdot \exp \left(-\frac{(i-\xi)^{2}+(j-\eta)^{2}}{2 \sigma^{2}}\right)
$$

where $i$ and $j$ are the varying pixel indices.

The detector position of the maximum $(\xi, \eta)$ is determined to subpixel level. The flux (total number of counts) $\Phi$ is estimated by $\Phi=2 \pi \cdot A \cdot \sigma^{2}$. This is an approximation, but it has the advantage that it also works when part of the PSF is outside the detector segment and therefore missing from the measurement.

The property values can only be determined reliably if a large enough fraction of the PSF is located within the detector segment. In order to guarantee this, the properties of a PSF are only determined and used for generalizing the PSF if the pixel with the highest value (after noise suppression) is not on or outside a segment edge, but surrounded by pixels that are inside the segment on all sides.

The PSFs are very localized on the detector. Including all pixels in a detector segment in the fit procedure may reduce the accuracy of the fit result because the "empty" parts of the detector still contain noise. Therefore, only the area surrounding the maximum is taken into account. The number of pixels included needs to be larger for wide PSFs than for narrow ones. Therefore, the fit is done twice. In the first instance all pixels that are both within the segment and within a fixed $15 \times 15$ square of pixels $(13 \times 13 \mathrm{~mm})$ around the maximum are used. The result of the first fit is used to obtain an approximate value for $\sigma$ which is subsequently used to determine which pixels are taken into account for the second fit: a square area of $2 \sigma \times 2 \sigma$, rounded off to an odd-valued integer, and centered around the maximum.

For each detector segment, four 3-D arrays are created where the values of the PSF properties are stored as a function of the $x, y, z$ coordinates of the measurement grid in the SPECT system. These arrays are from here on referred to as "property volumes."

\section{Generalization of the PSF's Model Over the Object Space Using an Analytical Pinhole Model}

One would like to limit the number of measurement points to a few hundred. On the other hand, PSFs must be available for all points that can be observed by one or more pinholes. When the

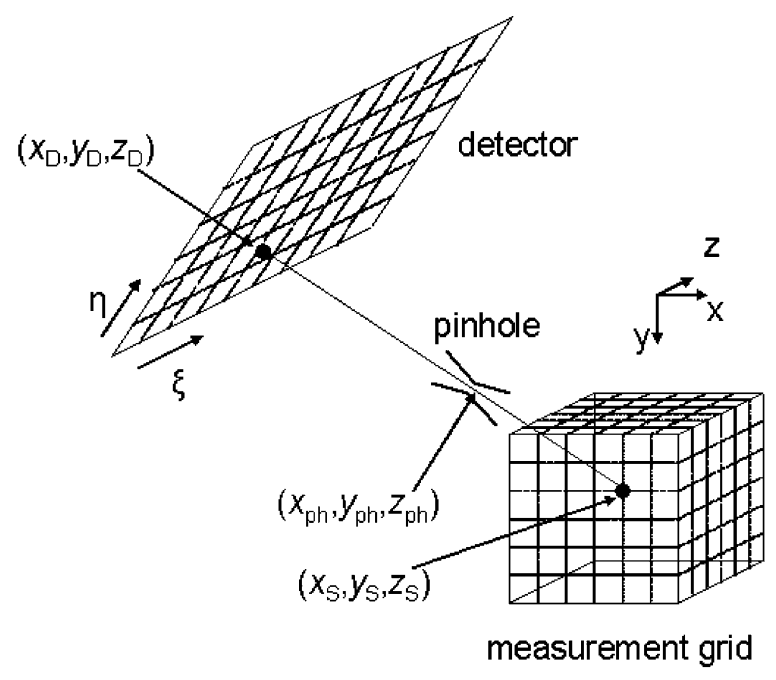

Fig. 5. Geometry of the model for the $\xi$ and $\eta$ position on the detector. A certain source location $\left(x_{\mathrm{S}}, y_{\mathrm{S}}, z_{\mathrm{S}}\right)$ is projected through the pinhole center onto a detector plane, the location of detection is at $x_{\mathrm{D}}, y_{\mathrm{D}}, z_{\mathrm{D}}$.

voxel size is $0.125 \mathrm{~mm}$, this means that fewer than one out of every 6000 voxels is a measurement position. The addition of a limited amount of geometrical information (see also Section IV discussion) is required.

1) Position of the PSF Maximum on the Detector: The geometry of the detector position model is illustrated in Fig. 5. The point-source activity is projected through the pinhole onto a detector segment of which the orientation is not precisely known. The effective position of detection is determined by the fit procedure which has three variables (the point source's position $\left.x_{\mathrm{S}}, y_{\mathrm{S}}, z_{\mathrm{S}}\right)$ and nine parameters: the pinhole's position $\left(x_{\mathrm{PH}}, y_{\mathrm{PH}}, z_{\mathrm{PH}}\right)$, the position $\left(x_{\mathrm{T}}, y_{\mathrm{T}}, z_{\mathrm{T}}\right)$ of the origin of the detector's coordinate system expressed in the coordinate system of the measurement grid, and three rotation or Euler angles $(\phi, \theta, \psi)$ for the detector orientation.

In order to build a mathematical model suitable for fitting, the available measured quantities need to be linked to the model's parameters and variables. The position of the point of detection $\left(x_{\mathrm{D}}, y_{\mathrm{D}}, z_{\mathrm{D}}\right)$ in the measurement grid's coordinate system can be related to the pinhole position $\left(x_{\mathrm{PH}}, y_{\mathrm{PH}}, z_{\mathrm{PH}}\right)$ and the source position $\left(x_{\mathrm{S}}, y_{\mathrm{S}}, z_{\mathrm{S}}\right)$ by assuming that the detection point, the center of the pinhole and the source are situated on the same line

$$
\frac{x_{\mathrm{S}}-x_{\mathrm{PH}}}{x_{\mathrm{PH}}-x_{\mathrm{D}}}=\frac{y_{\mathrm{S}}-y_{\mathrm{PH}}}{y_{\mathrm{PH}}-y_{\mathrm{D}}}=\frac{z_{\mathrm{S}}-z_{\mathrm{PH}}}{z_{\mathrm{PH}}-z_{\mathrm{D}}} .
$$

The coordinates $\left(x_{\mathrm{D}}, y_{\mathrm{D}}, z_{\mathrm{D}}\right)$ in the measurement grid's coordinate system of the point of detection are also related to the coordinates of this point in the detector's coordinate system $(\xi, \eta)$ via translation and rotation

$$
R \cdot\left(\begin{array}{c}
\xi \\
\eta \\
0
\end{array}\right)+\left(\begin{array}{l}
x_{\mathrm{T}} \\
y_{\mathrm{T}} \\
z_{\mathrm{T}}
\end{array}\right)=\left(\begin{array}{c}
x_{\mathrm{D}} \\
y_{\mathrm{D}} \\
z_{\mathrm{D}}
\end{array}\right)
$$

where $R$ is a standard rotation matrix expressed in terms of the Euler angles $\phi, \theta$, and $\psi$. Note that equating the third coordinate of the detection point in the detector's coordinate system to zero effectively means that all detections are in a plane; i.e., 


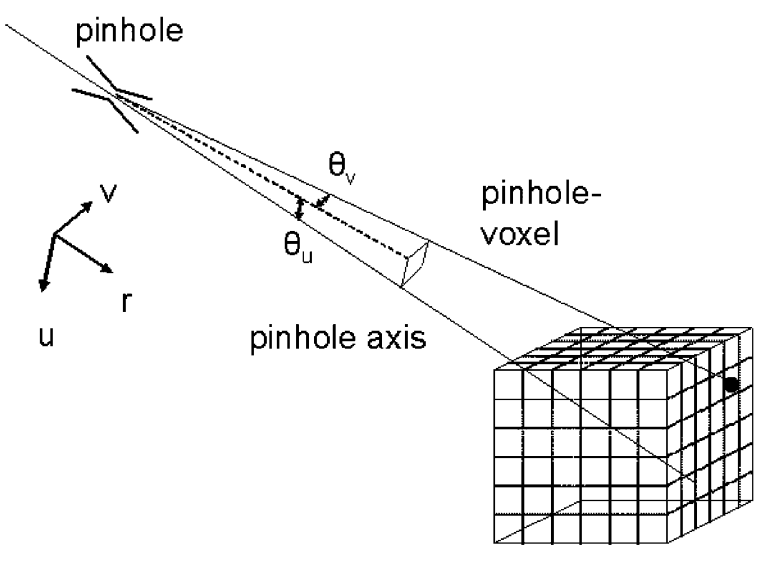

measurement grid

Fig. 6. Geometry for modeling the flux

the model approximates the detection volume in the scintillation crystal, which has a finite thickness and is expected to be slightly curved due to depth-of-interaction effects, by a single plane.

By combining (1) and (2) and eliminating $x_{\mathrm{D}}, y_{\mathrm{D}}$, and $z_{\mathrm{D}}$ it is possible to express the available data ( $\xi$ and $\eta$ of each pointsource projecting through the pinhole of this mini-camera) in terms of the model's variables and parameters. This is the desired form of a mathematical model in order to be suitable for parameter estimation by fitting. Fitting was performed using the Levenberg-Marquardt algorithm. The initial guess can be obtained by assuming that the pinhole location is on the surface of a cylinder, as seen from the center in the direction of the measurement grid position where the measured intensity is highest. For the initial guess of the detector's position and orientation, the detector can be assumed to be behind the pinhole, perpendicular to the pinhole axis at the average of the minimum and maximum pinhole-to-detector distances that occur in the U-SPECT-I system.

2) Flux Model: Like the position of the center of the PSF, the flux is also generalized using a parametric model and the same nonlinear fit routine. The geometry explaining the flux model is illustrated in Fig. 6. A coordinate system is defined with one axis (denoted "r") approximately along the pinhole axis. To find it without specifying the pinhole axis explicitely, we use the fact that the axis must go through the center of the pinhole as found in the position fit. The axis is assumed to go through the center of gravity of the cloud of measurement points as well. The other two axes (denoted " $u$ " and "v") are orthogonal to the pinhole axis and to each other. The flux $\Phi$ in a certain voxel is modeled

$\Phi=\frac{1}{r^{2}}\left(A+B \cdot \theta_{u}+C \cdot \theta_{v}+D \cdot \theta_{u}^{2}+E \cdot \theta_{v}^{2}+F \cdot \theta_{u} \cdot \theta_{v}\right)$

where $r$ is the component along the pinhole axis of the distance between the voxel and the pinhole position, obtained from the position fit carried out previously, and $\theta_{u}$ and $\theta_{v}$ are the distances from the voxel to the pinhole in the $\mathrm{u}$ and $\mathrm{v}$ directions, expressed as angles.
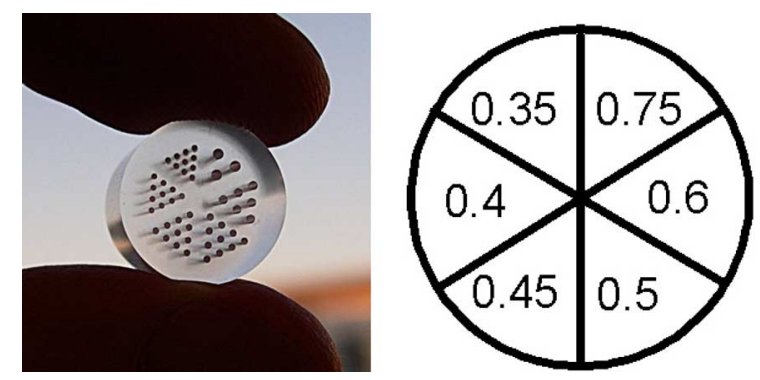

Fig. 7. Photograph and drawing of the capillary hot-rod phantom used in the experiments. Minimum distance between capillaries is equal to the diameter. Capillary sizes of this phantom range from 0.35 to $0.75 \mathrm{~mm}$.

Perpendicular to the pinhole axis, the flux is modeled by the general paraboloid of (3). If $\theta$ is the angle between the pinhole axis and the line connecting the voxel and the pinhole center, then one might expect a $\cos ^{3} \theta$ dependency. However, the projection data have been divided by the projection of an extended homogeneous source before the flux was measured so the flux data are not exactly described by the $\cos ^{3} \theta$ falloff away from the pinhole axis. The falloff is in fact still very smooth, however, and can be approximated by a paraboloid.

Using fitted parameter values $A-F$ the flux value can be calculated for each voxel in the object space.

3) Width: The width $\sigma$ of each PSF is described as

$$
\sigma=\sqrt{\left(D_{\mathrm{eff}, \mathrm{R}} \cdot \frac{l+r}{r}\right)^{2}+R^{2}}
$$

where $D_{\text {eff,R }}$ describes the effective pinhole diameter with respect to resolution, $l$ is the distance from the pinhole to the detector, projected along the pinhole axis, and $r$ is the distance from the point source to the pinhole, projected along the pinhole axis. The first term under the square root describes the effective pinhole diameter, projected magnified onto the detector as seen from the source. The second term $R$ describes the camera's intrinsic resolution. Again, the pinhole position is taken from the position fit and the axis is assumed to go through the center of gravity of the cloud of measurement points. $D_{\mathrm{eff}, \mathrm{R}}, R$, and $l$ are used as parameters in a fit using the Levenberg-Marquardt algorithm. Once their value has been determined for the current mini-camera (using $r$ and $\sigma$ as data), the width $\sigma$ can be calculated for each voxel in the object space.

\section{Calculation of the Supplementary PSFs for Storage in Tables}

Since a low-noise (by means of the noise suppression described in Section II-A) version of measured PSFs is available, we choose to use those measured PSFs to get the correct overall shape. To estimate a "missing" PSF (for a voxel location not in the measurement grid), the measured PSF whose corresponding point source location is nearest to the missing voxel location is used. That PSF is displaced on the detector to the location specified by the $\xi$ and $\eta$ values in the property volumes at the missing voxel location. The PSF is then stretched or contracted using the 
measured
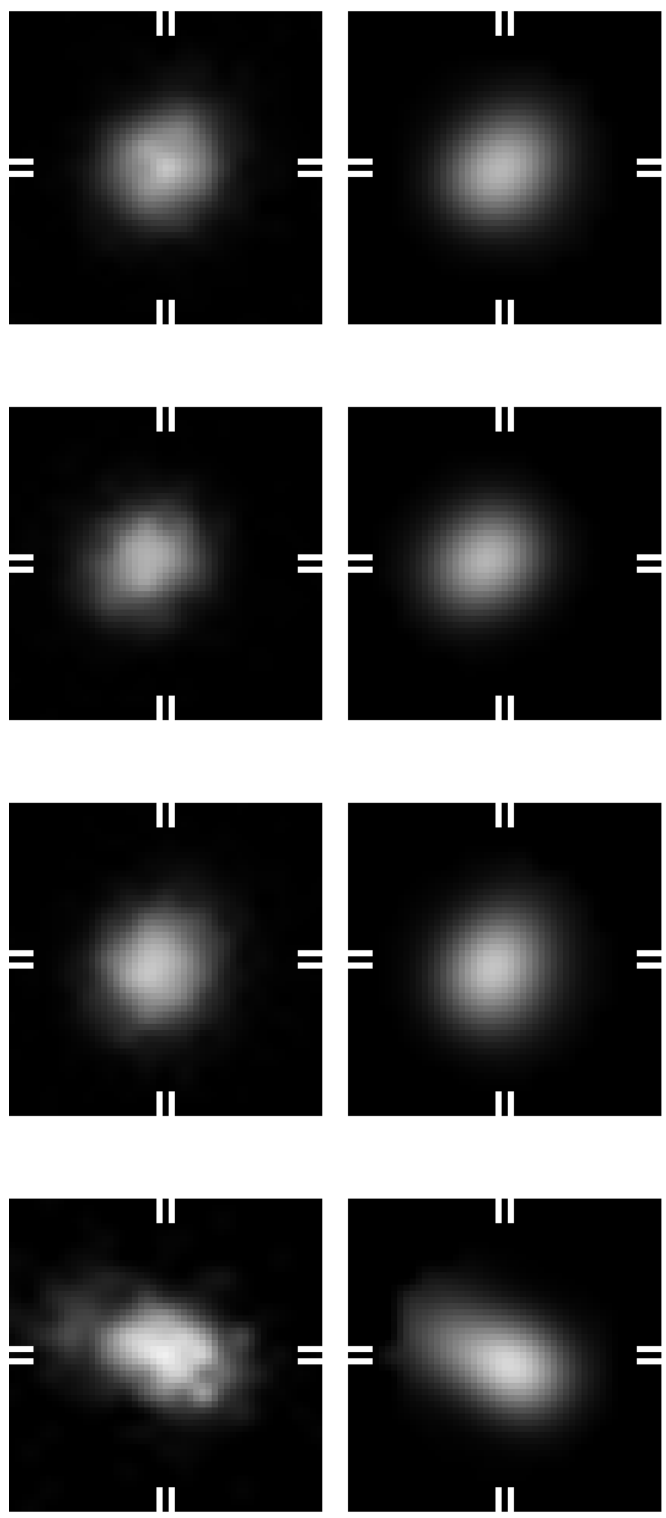

horizontal
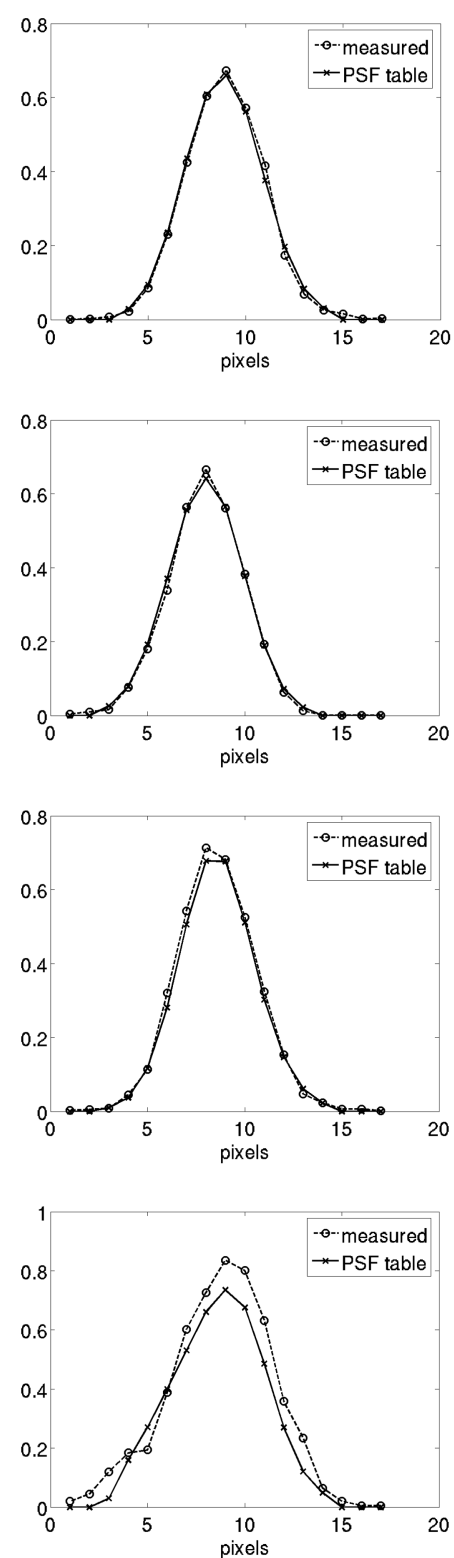

vertical
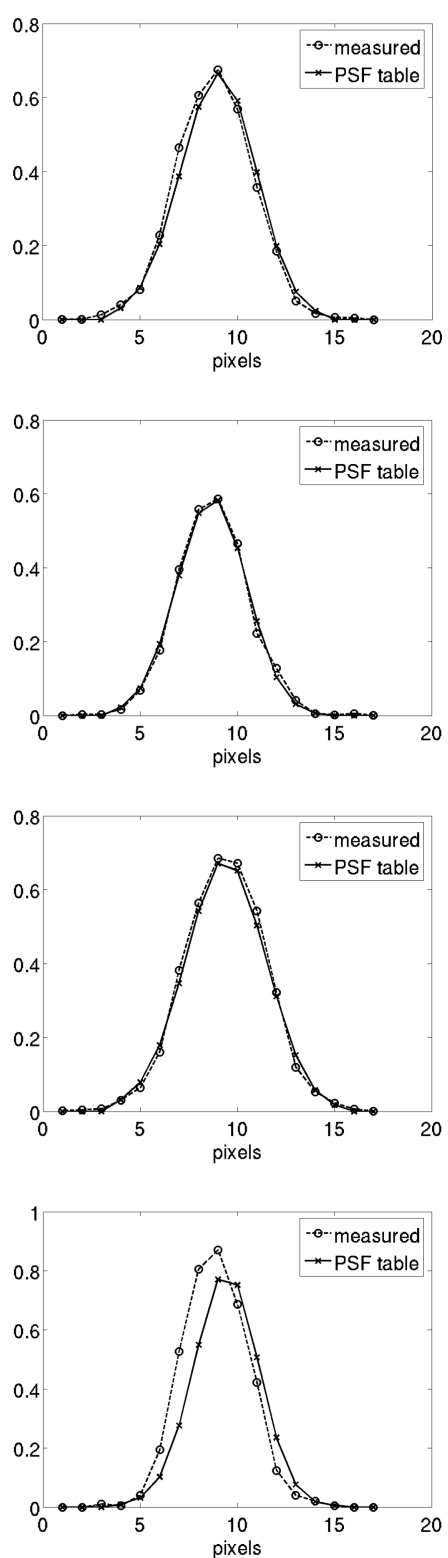

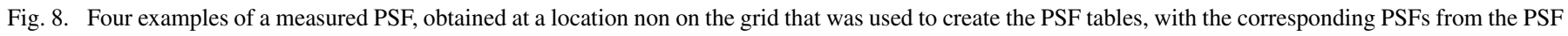
tables. First three examples are from the central area, where the PSFs from the PSF tables predict the position and shape very well. Last example was obtained at a

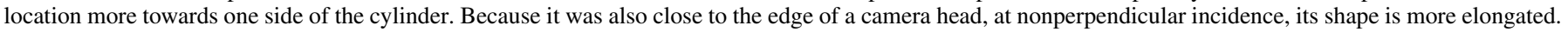

ratio of $\sigma$ at the missing point source position to $\sigma$ of the measured PSF in both directions. The number of pixels incorporated in the newly created PSF can be varied. We define the "tail size" to be the diameter of the PSF on the detector, where nonzero pixel values are created. It is expressed as the number of times that the FWHM of the PSF fits in this diameter. Finally, all pixel intensities in the newly created PSF are scaled such that their sum equals the flux specified by the flux volume at the missing point source location. After the PSF has been created in this way, the pixel intensities should be multiplied by the extended homogeneous source measurement to undo the effect of the division by this measurement at the stage where the properties were determined. According to this procedure, a PSF is calculated for all missing point source positions. These are stored on disk to be used in the image reconstruction algorithm.

\section{E. Validation}

We have validated the proposed generalization of PSFs by measuring some additional PSFs to the ones used to generate the PSF tables. These are compared to the corresponding PSFs in the PSF tables. The system is calibrated using 679 measurement positions, indicated by solid circles in Fig. 1. The spacing in the central part is $3 \mathrm{~mm}$ in the $x$ and $y$ (transaxial) directions and $1.5 \mathrm{~mm}$ in the $z$ (axial) direction. The outer positions are at $x$ or $y=13.5 \mathrm{~mm}$, spaced $4.5 \mathrm{~mm}$ in the $x, y$ plane and $3 \mathrm{~mm}$ in the $z$-direction. 


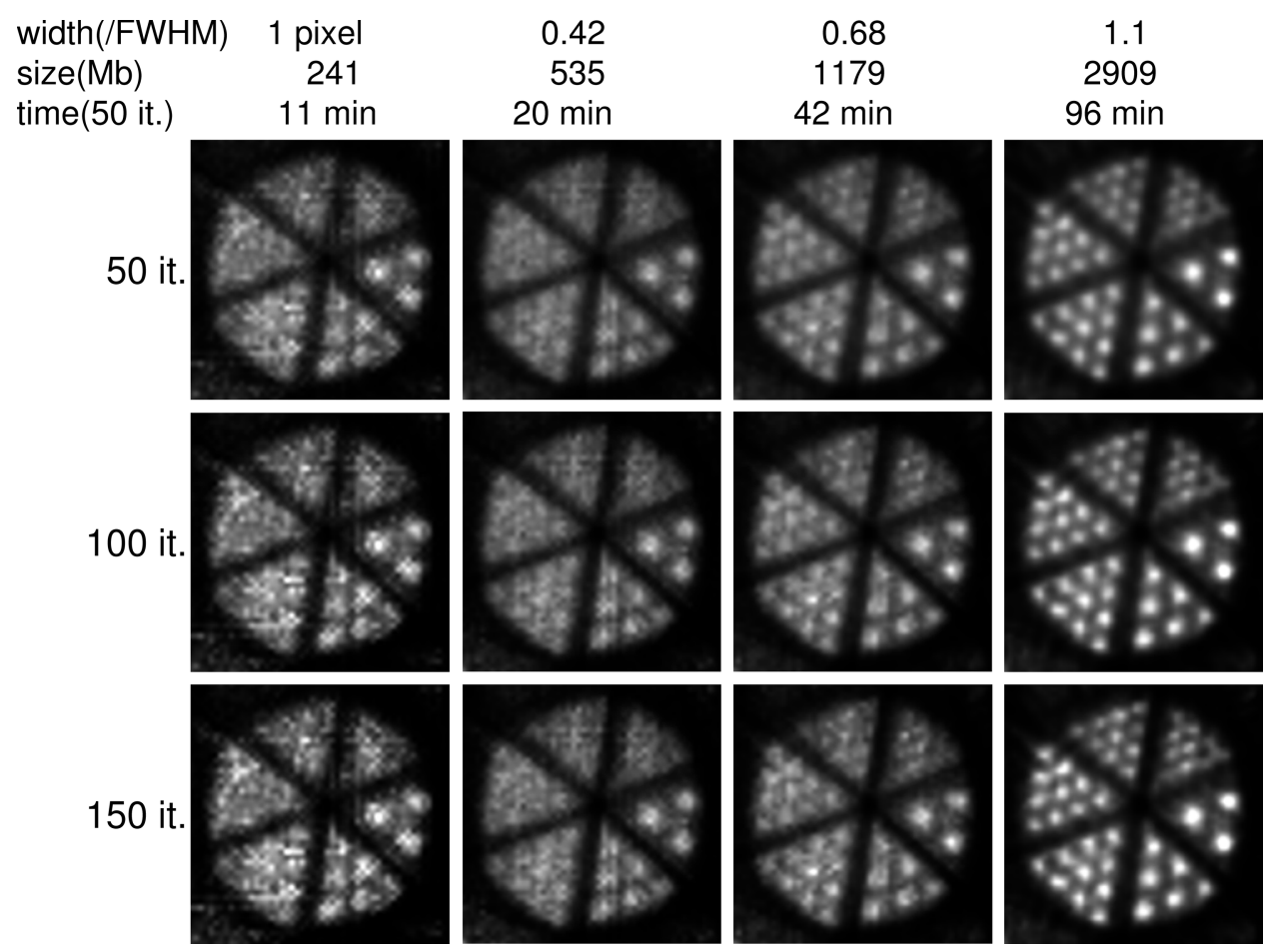

Fig. 9. Reconstruction for different PSF tail sizes obtained with 0.3-mm pinholes. Voxel size $0.1875 \mathrm{~mm}$. Slice thickness $0.375 \mathrm{~mm}$. Numbers at the top represent the full width of the truncated PSF expressed in units of its FWHM, the resulting matrix size on disk in megabytes, and an example reconstruction time for 50 iterations. Vertically the number of iterations is varied: $50,100,150$. Phantom capillary sizes are $0.75,0.6,0.5,0.45,0.4$, and $0.35 \mathrm{~mm}$.

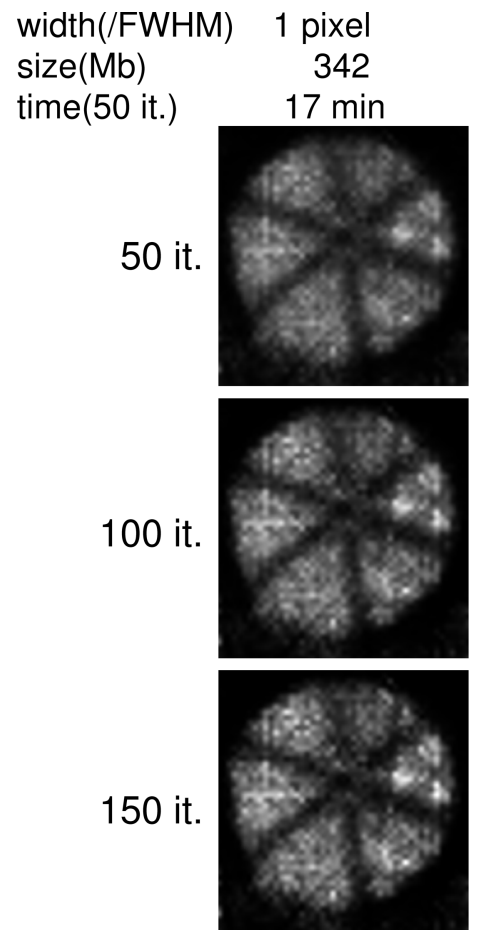

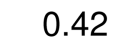

1031

$41 \mathrm{~min}$
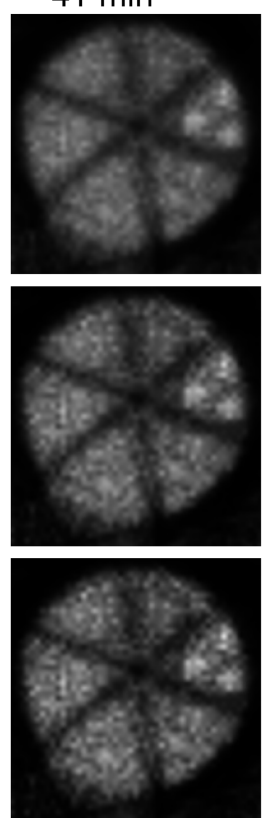
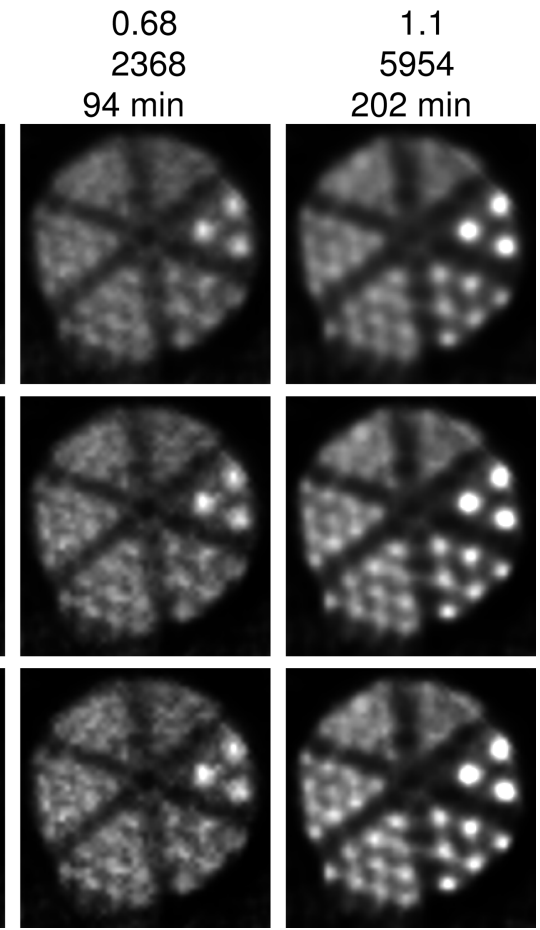

Fig. 10. Like Fig. 9, but for $0.6-\mathrm{mm}$ pinholes. Voxel size is $0.1875 \mathrm{~mm}$.

Another validation approach is to verify that the calibration method as a whole produces high resolution reconstructed images, which will also be described in the next section, using a capillary mini-Derenzo phantom, as shown in Fig. 7 as the object.

\section{RESULTS}

\section{A. Validation in the Projection Domain}

Fig. 8 presents some examples of PSFs from the PSF tables generated by the procedure, alongside experimentally measured 


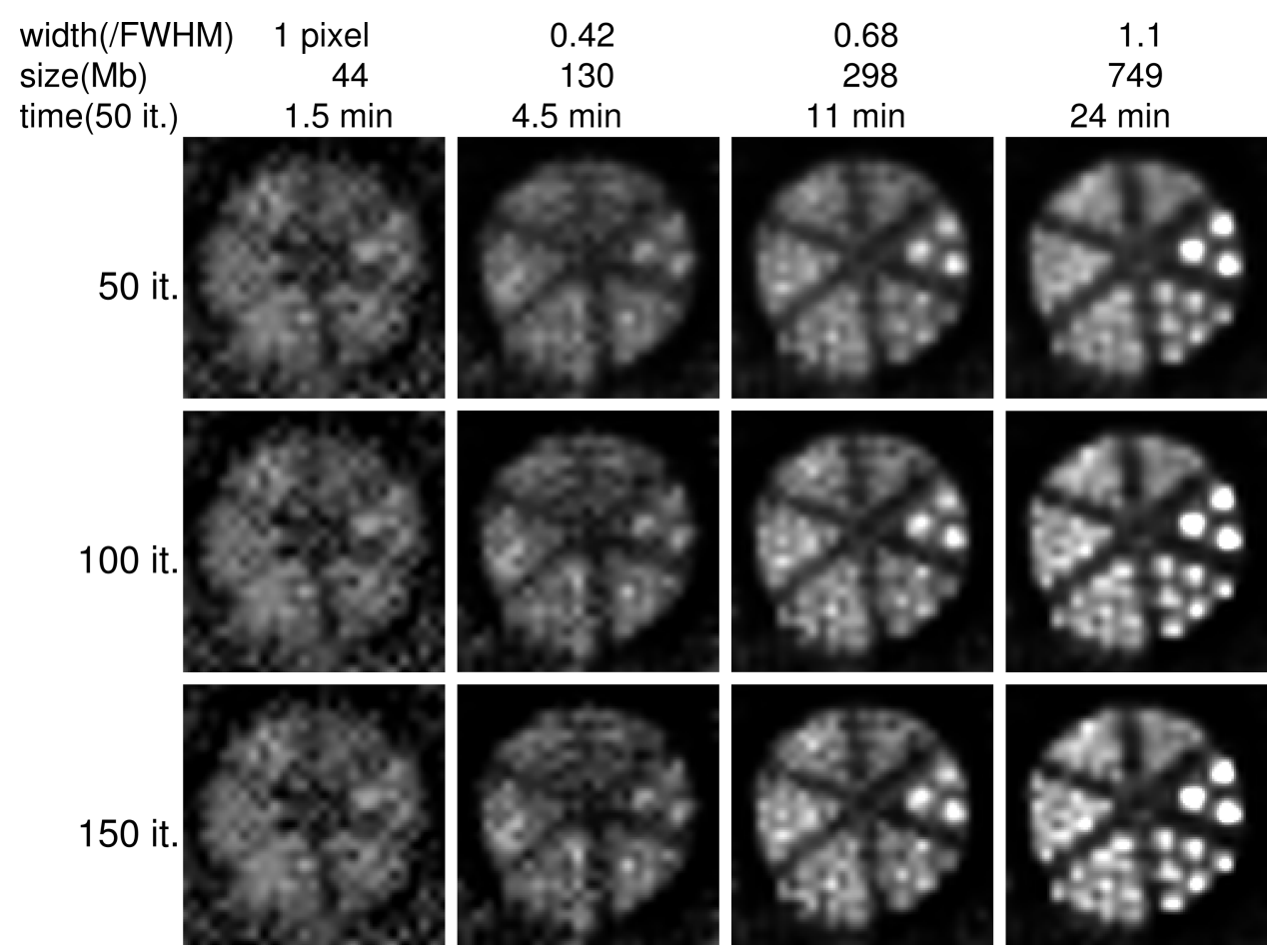

Fig. 11. Like Fig. 10, but for $0.375 \mathrm{~mm}$ voxel size. Slice thickness is also $0.375 \mathrm{~mm}$.

PSFs at the corresponding voxel location. Horizontal and vertical profiles through the center are shown that are summed over three pixels. The point source was at $x, y, z=(0,1.5,0) \mathrm{mm}$ in the first example, at $(1.5,0,0) \mathrm{mm}$ for the second and third example, and at $(13.5,9,1.5) \mathrm{mm}$ for the fourth example. The latter projects to a segment near to the edge of the detector. The first three examples show that in the central field-of-view area, the volume seen by all pinholes, there is a good agreement between the measured PSFs and the PSFs from the PSF tables. Therefore, the position, flux, and width are all correctly predicted by the model. The fourth example shows a small position mismatch in the vertical direction, on the order of $0.5 \mathrm{~mm}$ on the detector. This remains well below the detector's intrinsic resolution and would lead to a positioning error in the object space of less than $0.1 \mathrm{~mm}$. This example also shows an underestimation of the flux. This still leads to good reconstructions, as will be shown further on in this paper for small objects. Applications to objects larger than the central field-of-view are shown in [36].

\section{B. Reconstruction Quality Versus PSF Extent}

There is an important trade-off in reconstruction methods, including the iterative statistical method we use, between reconstruction accuracy and matrix size. To investigate one aspect of this trade-off, the "tail size" that was used when PSFs were created, was varied. The diameter of the detector area for which the PSF was created, was proportional to the generalized $\sigma$ of the PSF. The PSF generation is implemented in such a way that there is always at least one pixel in each PSF. For example, in the limiting case where the PSF diameter is $0 \times$ the FWHM ( $\delta$-response), all PSFs are created as the single pixel that is closest to the specified $\xi, \eta$ location, containing the total specified flux. Figs. 9-11 give reconstructed results for 50,100, and 150 iterations, while the tail size incorporated in the PSF is $0 \times$ FWHM, $0.43 \times$ FWHM, $0.68 \times$ FWHM, and $1.1 \times$ FWHM. Fig. 9 is for the $0.3-\mathrm{mm}$ pinholes and $0.1875 \mathrm{~mm}$ voxel size, Fig. 10 is for $0.6-\mathrm{mm}$ pinholes and $0.1875 \mathrm{~mm}$ voxel size, and Fig. 11 is for $0.6-\mathrm{mm}$ pinholes and $0.375 \mathrm{~mm}$ voxel size. The results are all compared at a slice thickness of $0.375 \mathrm{~mm}$. The resulting matrix sizes on disk are given for reference as well as example reconstruction times on a $2.66-\mathrm{GHz}$ Xeon system. This system had $8 \mathrm{~Gb}$ of memory, such that in all cases the matrix needed to be read from disk only once. The matrices used were available for a much larger voxel extent than the capillary phantom shown, which means that they would also be suitable for total-body mouse imaging [36] for example. The reconstruction times were all obtained with the same fairly straightforward implementation of the maximum likelihood expectation maximization (ML-EM) algorithm which was not optimized for speed. The results show that the largest PSF width of $1.1 \times$ FWHM resulted in the reconstruction that allows the best visual distinction of rods. Wider PSFs were also tested, but did not lead to better results.

Voxel size has a big impact on matrix size and thus reconstruction time, because of the cubic power relation between voxel size and matrix size. The effect of doubling the voxel size (leading to an eight-fold reduction in matrix size) on image quality for the case of $0.6-\mathrm{mm}$ pinholes can be seen by comparing Figs. 10 and 11. While the smaller voxel size results look better, the difference is not so dramatic that the coarser voxels are useless; in some applications the images with the coarser voxels may be considered good enough-estimating the total amount of activity in a fairly large region-of-interest over multiple points in time for example-where the gain in speed could make it worthwhile to use coarse voxels. 


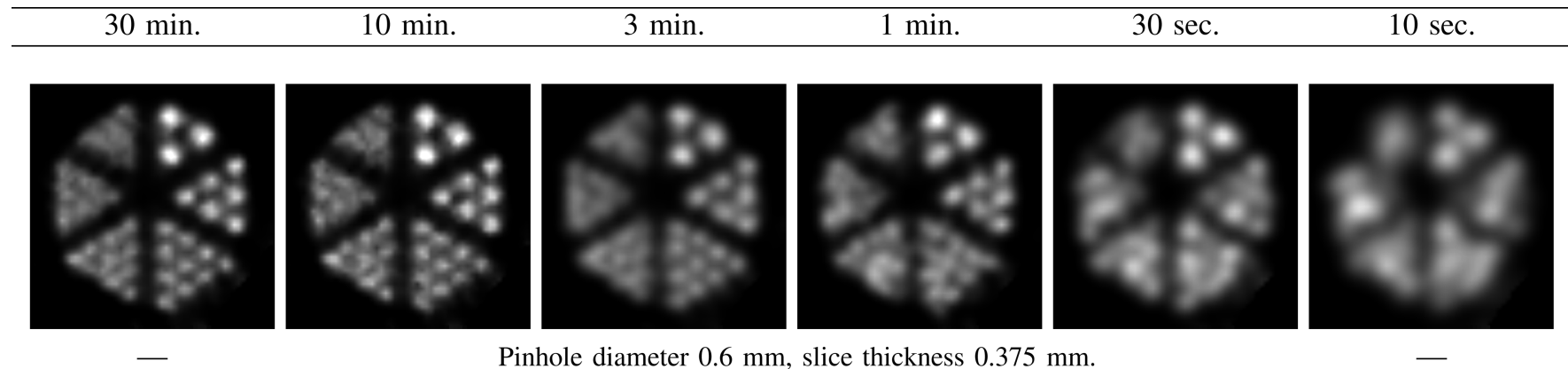

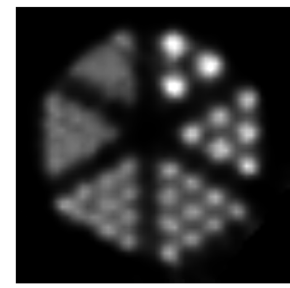

-
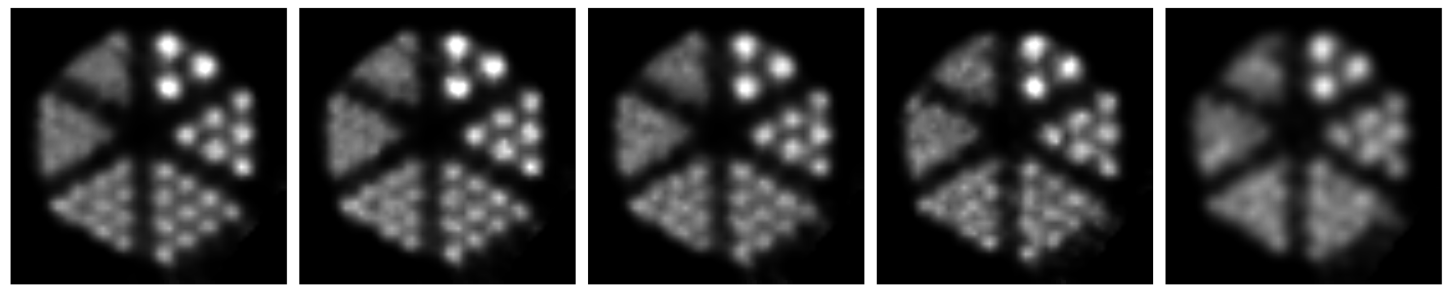

Pinhole diameter $0.6 \mathrm{~mm}$, slice thickness $3.75 \mathrm{~mm}$.
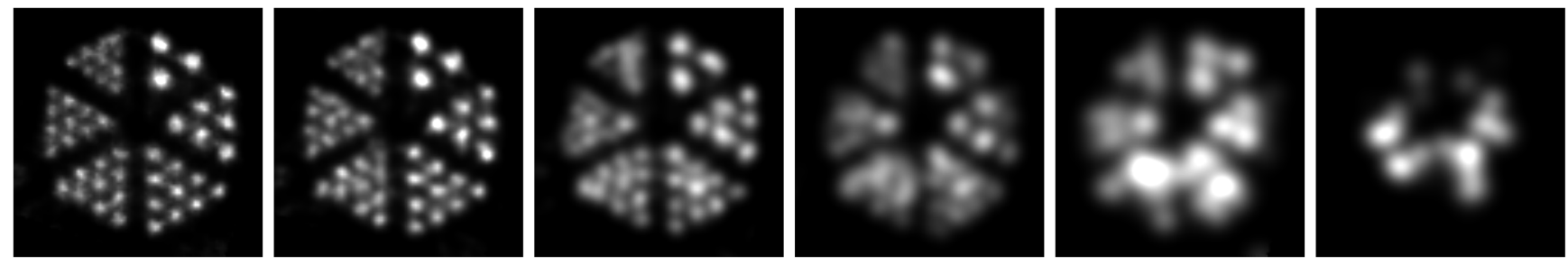

Pinhole diameter $0.3 \mathrm{~mm}$, slice thickness $0.375 \mathrm{~mm}$.

$-$

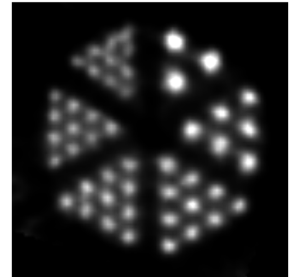

$-$
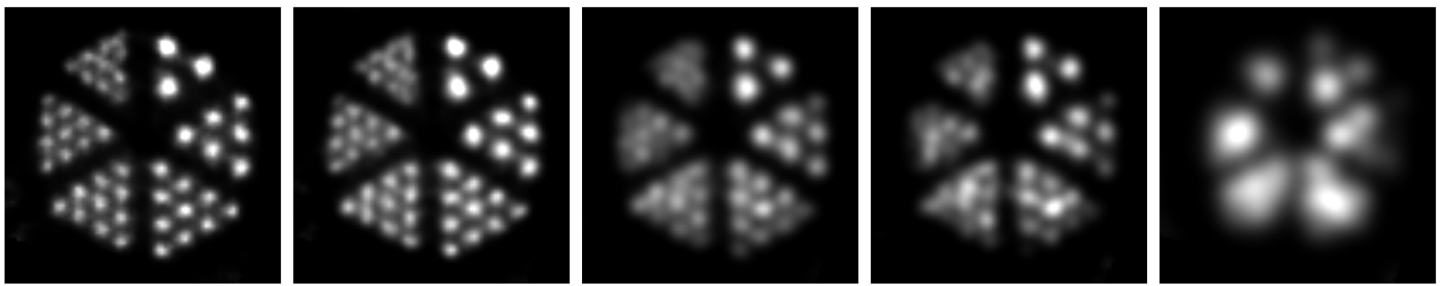

Pinhole diameter $0.3 \mathrm{~mm}$, slice thickness $3.75 \mathrm{~mm}$.

Fig. 12. Influence of acquisition time (total number of detected counts) on the reconstructed image. This panel shows that with 0.3 -mm pinholes a resolution of $<0.35 \mathrm{~mm}(0.04 \mu \mathrm{l})$ can be obtained. At extremely short acquisition times a resolution of $0.75 \mathrm{~mm}$ can still be reached. Acquisition times range from half an hour down to $10 \mathrm{~s}$.

With $0.3-\mathrm{mm}$ pinholes smaller rods can be distinguished than with the 0.6-mm pinholes, which may not be surprising. What may not be obvious a priori is that the $0.6-\mathrm{mm}$ pinholes actually require at least the same PSF size in units of FWHM as the $0.3-\mathrm{mm}$ pinholes in order to reach the highest achievable resolution, while each FWHM is already larger than the corresponding PSF from a 0.3-mm pinhole because of the larger diameter. This makes the $0.3-\mathrm{mm}$ pinholes matrix smaller than the 0.6-mm pinholes matrix, when comparing at equal voxel and pixel size, by a factor of approximately 2 in the given examples.

\section{Reconstruction With Different Acquisition Times for Two Pinhole Diameters}

Fig. 12 shows the hot-rod resolution phantom images compared for different acquisition times. The activity concentration was $300 \mathrm{MBq} / \mathrm{ml} \mathrm{Tc}-99 \mathrm{~m}$ (25 MBq in total in the capillaries). The acquisition times can of course be scaled if one wants to have an impression of the achievable resolution at other concentrations. The experiment was done with both the 0.3- and the $0.6-\mathrm{mm}$ pinholes and after reconstruction, the results are displayed for a slice thickness of $0.375 \mathrm{~mm}$ and $3.75 \mathrm{~mm}$ for each pinhole diameter. Visually the $0.3-\mathrm{mm}$ pinholes yield a superior resolution for acquisition times down to $1 \mathrm{~min}$ (or even shorter if the larger slice thickness is viewed). Only for extremely short acquisition times (below $1 \mathrm{~min}$ ) do the $0.6-\mathrm{mm}$ pinholes produce superior results, because the $0.3-\mathrm{mm}$ pinholes collect too few counts. For $10 \mathrm{~s}$ acquisition time, it is still possible to obtain $0.75 \mathrm{~mm}$ reconstructed resolution for the $0.6-\mathrm{mm}$ pinholes. The best achievable resolution, on the other hand, is seen to be $0.45 \mathrm{~mm}$ for the $0.6-\mathrm{mm}$ pinholes and below $0.35 \mathrm{~mm}$ for the 0.3-mm pinholes. 


\section{DISCUSSION}

In this paper, we have presented a method to obtain full PSF tables (i.e., calibrate a multipinhole system) based on a limited number of measurements and generalization of PSF properties over the object space. When using a scanning-focus method [36] to image a volume that is larger than the central field-of-view of the scanner, the PSFs need to be available throughout the collimator cylinder, including the outer area close behind the pinholes. Some geometrical information about the system needs to be added for a generalization algorithm to be able to correctly find especially the position on the detector of each PSF. Therefore, we have added some geometrical information in our model, but not as much as specifying the exact location, orientation and shape of every pinhole. The uncertainty in those is one of the reasons to use experimentally determined PSFs in the first place. Trying to find a balance between adding too little and too much geometrical information we have arrived at the parametric fit procedure described in Section II-C.

The required accuracy of the whole process also puts some demands on the mechanical accuracy of the robotic XYZ-stage that is used to step the point source through the collimator. The most important requirement of its accuracy and alignment is that the relative positions of the grid of points used to calibrate the system, are accurate to subvoxel level. The absolute position of the grid as a whole and its alignment with respect to the collimator are important to a lesser degree.

The system matrix is determined experimentally (as opposed to, e.g., analytically calculated) because this automatically takes into account most physical effects that influence detection. Wide-angle scatter is not incorporated in the PSF tables. However, the effects of object attenuation and scatter are much less severe in small-animal imaging as in human imaging. Pinhole aperture scattering typically amounts to a few percent of the total detected counts when Tc-99m is used [44].

The example reconstruction times that were indicated, were obtained with a straightforward implementation of the ML-EM algorithm. By using block-iterative algorithms (e.g., orderedsubsets EM) together with faster processors with parallel processing, the reconstruction can be accelerated by more than two orders of magnitude. Since it is not always given that OS-EM methods will converge in the same way as ML-EM, and the way to optimally implement them for a system such as U-SPECT is a topic of investigation in itself, we chose to use a "clean" ML-EM algorithm for the comparisons and results in this paper.

\section{CONCLUSION}

This paper presents a method to create full PSF tables for a small-animal SPECT scanner from a limited number of measurements with a point source. It is based on determining four properties of a PSF and generalizing those properties to include all locations where no point-source measurement is available. In this way the measurement of the PSF for all individual voxels is unnecessary. The full shape of measured PSFs can be used in generating full PSF tables (the system matrix). It is shown that the method correctly estimates the PSF at a "missing" location in a number of examples and leads to PSF tables that give sub-half-millimeter reconstruction results.
Using 0.6-mm pinholes, it is possible to combine subminute acquisition times with submillimeter resolution. PSF tables that model the actual system more accurately, e.g., by incorporating more of the tails, lead to better images at the cost of system matrix size.

\section{ACKNOWLEDGMENT}

The authors would like to thank Dr. L. R. Furenlid (University of Arizona) for his advice on producing the point sources used for calibration.

\section{REFERENCES}

[1] K. Ishizu, T. Mukai, Y. Yonekura, M. Pagani, T. Fujita, Y. Magata, S. Nishizawa, N. Tamaki, H. Shibasaki, and J. Konishi, "Ultra-high resolution SPECT system using four pinhole collimators for small animal studies," J. Nucl. Med., vol. 36, pp. 2282-2287, 1995.

[2] S. R. Meikle, R. R. Fulton, S. Eberl, M. Dahlbom, K. P. Wong, and M. J. Fulham, "An investigation of coded aperture imaging for small animal SPECT,” IEEE Trans. Nucl. Sci., vol. 48, pp. 816-821, Jun. 2001.

[3] D. P. McElroy, L. R. MacDonald, F. J. Beekman, Y. C. Wang, B. E. Patt, J. S. Iwanczyk, B. M. W. Tsui, and E. J. Hoffman, "Performance evaluation of A-SPECT: A high resolution desktop pinhole SPECT system for imaging small animals," IEEE Trans. Nucl. Sci., vol. 49, no. 5, pp. 2139-2147, Oct. 2002.

[4] S. D. Metzler, R. J. Jaszczak, N. H. Patil, S. Vemulapalli, G. Akabani, and B. B. Chin, "Molecular imaging of small animals with a triple-head SPECT system using pinhole collimation," IEEE Trans. Med. Imag., vol. 24 , no. 7 , pp. 853-862, Jul. 2005.

[5] N. U. Schramm, G. Ebel, U. Engeland, T. Schurrat, M. Béhé, and T. M. Behr, "High-resolution SPECT using multipinhole collimation," IEEE Trans. Nucl. Sci., vol. 50, no. 3, pp. 315-320, Jun. 2003.

[6] C. Lackas, N. U. Schramm, J. W. Hoppin, U. Engeland, A. Wirrwar, and H. Halling, "T-SPECT: A novel imaging technique for small animal research," IEEE Trans. Nucl. Sci., vol. 52, no. 1, pp. 181-187, Feb. 2005.

[7] A. L. Goertzen, D. W. Jones, J. Seidel, K. Li, and M. V. Green, "First results from the high-resolution mousespect annular scintillation camera," IEEE Trans. Med. Imag., vol. 24, no. 7, pp. 863-867, Jul. 2005.

[8] S. R. Meikle, P. Kench, M. Kassiou, and R. B. Banati, "Small animal SPECT and its place in the matrix of molecular imaging technologies," Phys. Med. Biol., vol. 50, pp. R45-R61, 2005.

[9] K. Peremans, B. Cornelissen, B. Van Den Bossche, K. Audenaert, and C. Van de Wiele, "A review of small animal imaging, planar and pinhole spect gamma camera imaging," Veterinary Radiol. Ultrasound, vol. 46, no. 2, pp. 162-170, 2005.

[10] B. M. W. Tsui and Y. C. Wang, "High-resolution molecular imaging techniques for cardiovascular research," J. Nucl. Cardiol., vol. 12, no. 3, pp. 261-267, 2005.

[11] F. J. Beekman and F. van der Have, "The pinhole: Gateway to ultrahigh resolution three-dimensional radio-nuclide imaging," Eur. J. Nucl. Med. Molec. Imag., vol. 34, no. 2, pp. 151-161, 2007.

[12] K. Vunckx, D. Bequé, M. Defrise, and J. Nuyts, "Single and multipinhole collimator design evaluation method for small animal SPECT," IEEE Trans. Med. Imag., vol. 27, no. 1, pp. 36-46, Jan. 2008.

[13] R. K. Rowe, J. N. Aarsvold, H. H. Barrett, J. C. Chen, W. P. Klein, B. A. Moore, I. W. Pang, D. D. Patton, and T. A. White, "A stationary hemispherical SPECT imager for three-dimensional brain imaging," $J$. Nucl. Med., vol. 34, pp. 474-480, 1993.

[14] Z. Liu, G. A. Kastis, G. D. Stevenson, H. H. Barrett, L. R. Furenlid, M. A. Kupinski, D. D. Patton, and D. W. Wilson, "Quantitative analysis of acute myocardial infarct in rat hearts with ischemia-reperfusion using a high-resolution stationary SPECT system," J. Nucl. Med., vol. 43, no. 7, pp. 933-939, 2002.

[15] L. R. Furenlid, D. W. Wilson, C. Yi-chun, K. Hyunki, P. J. Pietraski, M. J. Crawford, and H. H. Barrett, "FastSPECT II: A second-generation high-resolution dynamic SPECT imager," IEEE Trans. Nucl. Sci., vol. 51, no. 3, pp. 631-635, Jun. 2004.

[16] F. J. Beekman, F. van der Have, B. Vastenhouw, A. J. A. van der Linden, P. P. van Rijk, J. P. H. Burbach, and M. P. Smidt, "U-SPECT-I: A novel system for submillimeter-resolution tomography with radiolabeled molecules in mice," J. Nucl. Med., vol. 46, pp. 1194-1200, 2005. 
[17] F. J. Beekman and B. Vastenhouw, "Design and simulation of a highresolution stationary SPECT system for small animals," Phys. Med. Biol., vol. 49, pp. 4579-4592, 2004.

[18] G. K. Kastis, H. B. Barber, H. H. Barrett, H. C. Gifford, I. W. Pang, D. D. Patton, G. Stevenson, and D. W. Wilson, "Comparison of fluorine-18-fdg PET and thallium-201 SPECT in evalution of lung cancer," J. Nucl. Med., vol. 39, pp. 9-15, 1998.

[19] B. Vastenhouw, F. van der Have, A. J. A. van der Linden, L. von Oerthel, J. Booij, J. P. H. Burbach, M. P. Smidt, and F. J. Beekman, "Movies of dopamine transporter occupancy with ultra-high resolution focusing pinhole SPECT," Molecular Psychiatry, vol. 12, pp. 984-987, 2007.

[20] E. P. Ficaro, J. A. Fessler, P. D. Shreve, J. N. Kritzman, P. A. Rose, and J. R. Corbett, "Simultaneous transmission/emission myocardial perfusion tomography: Diagnostic-Accuracy of attenuation corrected tc-99m sestamibi SPECT," Circulation, vol. 93, pp. 463-473, 1996.

[21] F. J. Beekman, C. Kamphuis, and E. C. Frey, "Scatter compensation methods in 3-D iterative SPECT reconstruction: A simulation study," Phys. Med. Biol., vol. 42, no. 8, pp. 1619-1632, Aug. 1997.

[22] C. E. Floyd, R. J. Jaszczak, K. L. Greer, and R. E. Coleman, "Inverse Monte Carlo as a unified reconstruction algorithm for ECT," J. Nucl. Med., vol. 27, no. 10, pp. 1577-1585, 1986.

[23] D. J. Kadrmas, E. C. Frey, S. S. Karimi, and B. M. W. Tsui, "Fast implementations of reconstruction-based scatter compensation in fully 3-D SPECT image reconstruction," Phys. Med. Biol., vol. 43, pp. 857-874, 1998.

[24] B. M. W. Tsui, E. C. Frey, K. J. LaCroix, D. S. Lalush, W. H. McCarthy, M. A. King, and G. T. Gullberg, "Quantitative myocardial perfusion SPECT*1," J. Nucl. Cardiol., vol. 5, no. 5, pp. 507-522, Sep. 1998.

[25] S. D. Metzler, J. E. Bowsher, K. L. Greer, and R. J. Jaszczak, "Analytic determination of the pinhole collimator's point-spread function and RMS resolution with penetration," IEEE Trans. Med. Imag., vol. 21, no. 8, pp. 878-887, Aug. 2002.

[26] D. Bequé, J. Nuyts, G. Bormans, P. Suetens, and P. Dupont, "Characterization of pinhole SPECT acquisition geometry," IEEE Trans. Nucl. Sci., vol. 22, no. 5, pp. 599-612, May 2003.

[27] R. Accorsi and S. D. Metzler, "Analytic determination of the resolution-equivalent effective diameter of a pinhole collimator," IEEE Trans. Med. Imag., vol. 23, no. 6, pp. 750-763, Jun. 2004.

[28] J. Y. Li, R. J. Jaszczak, H. L. Wang, K. L. Greer, and R. E. Coleman, "Determination of both mechanical and electronic shifts in cone-beam SPECT," Phys. Med. Biol., vol. 38, pp. 743-754, 1993.

[29] P. Rizo, P. Grangeat, and R. Guillemaud, "Geometric calibration method for multiple-head cone-beam SPECT system," IEEE Trans. Nucl. Sci., vol. 41, no. 6, pp. 2748-2757, Dec. 1994.

[30] F. Noo, R. Clackdoyle, C. Mennessier, T. A. White, and T. J. Roney, "Analytic method based on identification of ellipse parameters for scanner calibration in cone-beam tomography," Phys. Med. Biol., vol. 45 , pp. 3489-3508, 2000.
[31] D. Bequé, J. Nuyts, P. Suetens, and G. Bormans, "Optimization of geometrical calibration in pinhole SPECT," IEEE Trans. Med. Imag., vol. 24, no. 2, pp. 180-190, Feb. 2005.

[32] S. D. Metzler, R. J. Jaszczak, N. H. Patil, S. Vemulapalli, G. Akabani, and B. B. Chin, "Determination of mechanical and electronic shifts for pinhole SPECT using a single point source," IEEE Trans. Med. Imag., vol. 24, no. 3, pp. 361-370, Mar. 2005.

[33] S. D. Metzler and R. J. Jaszczak, "Simultaneous multi-head calibration for pinhole SPECT," IEEE Trans. Nucl. Sci., vol. 53, no. 1, pp. 113-120, 2006.

[34] Y.-C. Chen, L. R. Furenlid, D. W. Wilson, and H. H. Barrett, Small-Animal SPECT Imaging. New York: Springer Science Business Media, 2005, ch. 12, pp. 195-201.

[35] J. Y. Hesterman, M. A. Kupinski, L. R. Furenlid, D. W. Wilson, and H. H. Barrett, "The multi-module, multi-resolution system (M3R): A novel small-animal SPECT system," Med. Phys., vol. 34, no. 3, pp. 987-993, 2007.

[36] B. Vastenhouw and F. J. Beekman, "Submillimeter total-body murine imaging with U-SPECT-I,” J. Nucl. Med., vol. 48, no. 3, pp. 487-493, 2007.

[37] F. van der Have, B. Vastenhouw, M. Rentmeester, and F. J. Beekman, "System calibration and statistical image reconstruction for sub-mm stationary pinhole SPECT," in Conf. Rec. IEEE 2005 Nucl. Sci. Symp. Med. Imag. Conf., 2005, pp. M11-291.

[38] F. van der Have, B. Vastenhouw, and F. J. Beekman, "U-SPECT-II: A versatile sub-half-mm resolution small animal SPECT system," presented at the Soc. Nucl. Med. Conf., Washington D.C., 2007.

[39] F. J. Beekman, A method for obtaining a tomographic image, including an apparatus PCT-application WO02093195, May 2002.

[40] F. J. Beekman, "Werkwijze voor het verkrijgen van een tomografische afbeelding, alsmede een inrichting," Dutch patents 1018060 and 1019666 , U.S. patent US7145153 B2, May 11, 2001.

[41] W. H. Richardson, "Bayesian-based iterative method of image restoration," J. Opt. Soc. Amer., vol. 62, pp. 55-59, 1972.

[42] L. B. Lucy, "An iterative technique for the rectification of observed distributions," Astronom. J., vol. 79, no. 6, pp. 745-754, Jun. 1974.

[43] A. P. Colijn and F. J. Beekman, "Accelerated simulation of cone beam X-ray scatter projections," IEEE Trans. Med. Imag., vol. 23, no. 5, pp. 584-590, May 2004.

[44] F. van der Have and F. J. Beekman, "Photon penetration and scatter in micro-pinhole imaging: A Monte Carlo investigation," Phys. Med. Biol., vol. 49, pp. 1369-1386, 2004.

[45] W. H. Press, B. P. Flannerly, S. A. Teukolsky, and W. T. Vetterling, Numerical Recipes in C. Cambridge, U.K.: Cambridge Univ. Press, 1988. 